\title{
Frontiers Commentary on Tallet et al. Investigation of prolactin receptor activation and blockade using time-resolved fluorescence resonance energy transfer
}

\author{
Ralf Jockers ${ }^{1,2,3}$ * \\ 1 INSERM, U1016, Institut Cochin, Paris, France \\ ${ }^{2}$ CNRS UMR 8104, Paris, France \\ ${ }^{3}$ University Paris Descartes, Sorbonne Paris Cité, Paris, France \\ *Correspondence: ralf.jockers@inserm.fr
}

Edited and reviewed by:

Derek LeRoith, Mount Sinai School of Medicine, USA

Keywords: dimerization, TR-FRET, PRLR-I146L, antagonist, commentary

\section{A commentary on}

Investigation of prolactin receptor activation and blockade using time-resolved fluorescence resonance energy transfer by Tallet E, Fernandez I, Zhang C, Salsac M, Gregor N, Ayoub MA, et al. (2011). Front Endocrin 2:29. doi:10.3389/fendo. 2011.00029

Transmission of extracellular signals into the cell often relies on the binding of extracellular signaling molecules to membrane receptors. How membrane receptors transmit these signals into the cell and activate intracellular signaling cascades has been an intense field of research over the last 20 years. Basically, two competing models have been proposed. In the first model, binding of signaling molecules promotes ligand-induced dimerization/oligomerization of receptors. According to the second model, binding of signaling molecules triggers ligand-induced conformational changes within preformed dimeric/oligomeric receptors.

For many years, the model of ligandinduced dimerization was the preferred model to explain the activation of many single transmembrane-spanning receptors that rely on the (trans)phosphorylation of their intracellular domains. One of these receptors is the prolactin receptor (PRLR), which belongs to the cytokine receptor family that is devoid of intrinsic kinase activity but constitutively interacts with the JAK2 tyrosine kinase. PRLR activation triggers first trans-phosphorylation of two JAK2 molecules (each bound to one PRLR molecule), followed by receptor phosphorylation by JAK2 and recruitment of STAT5 molecules promoting further downstream signaling events.

Before the publication of the article of Tallet et al. in Frontiers in Endocrinology (1), conflicting results had been reported in the literature concerning the activation model of the PRLR. Indeed, Qazi et al., using bioluminescence resonance energy transfer (BRET) and co-immunoprecipitation (coIP) approaches, concluded that the PRLR constitutively homodimerize (or heterodimerized when long and short isoforms were co-expressed in the same cell) (2). A similar conclusion was reached in another study based on co-IP experiments further arguing for the existence of ligand-independent homodimers of human PRLR isoforms, with the transmembrane domain being the main dimer interface (3). Contrasting results were however reported in another BRET study with various C-terminally tagged PRLR isoforms. The authors revealed liganddependent changes in BRET signals that discriminated receptor agonists from a partial agonist/antagonist (4). Unfortunately, the authors did not perform complementary experiments to determine whether the BRET changes originated from ligandinduced dimerization or conformational changes as previously reported for other cytokine receptors (5).

The article of Tallet et al. published in Frontiers in Endocrinology made a decisive contribution because the authors recapitulated all previously employed techniques (co-IP, BRET) in a single study and went beyond the current status by exploiting further techniques such as blue native gel electrophoreses and time-resolved fluorescence resonance energy transfer (TRFRET). This presents the first application of the TR-FRET technology to cytokine receptors. TR-FRET is based on the energy transfer between the energy donor terbium cryptate $(\mathrm{Tb})$ and an energy acceptor. Due to the long-lived fluorescence properties of $\mathrm{Tb}$, measurement can be performed after a time delay of $50-150 \mu$ s to avoid interference with cellular autofluorescence, thereby considerably improving the signalto-noise ratio of the assay (6). TR-FRET has several interesting features that differentiate this technique from BRET. Whereas BRET signals typically represent all tagged proteins that interact, irrespective of their subcellular localization, TR-FRET signals are only generated by the fraction of proteins located at the plasma membrane. This difference seems to be important as the PRLR is mainly located inside the cell and only the fraction that reaches the cell surface becomes activated by the extracellular prolactin. Furthermore, the intensity of TR-FRET signals directly correlates with the distance between the donor and acceptor, which allows us to be conclusive regarding this parameter. This is more difficult to achieve with the BRET technique as BRET signals depend not only on the distance but also on the relative orientation of the BRET donor and acceptor. In the study of Tallet et al., the authors designed a TR-FRET assay to monitor putative ligand-induced TR-FRET signal changes between two extracellular PRLR domains. Taken all techniques together, the authors convincingly demonstrated that (at least part of) the PRLR population 
is pre-dimerized at the cell membrane and that activation (via ligand binding or I146L mutation that constitutively activates the receptor) or inactivation (by receptor antagonists) do not alter the number of dimers or the distance between the two extracellular domains of PRLR dimers, suggesting that the control of receptor activity involves subtle conformational changes.

A subsequent study that solved the nuclear magnetic resonance solution structure of the membrane proximal D2 domain of the human PRLR (7), confirmed this conclusion as the authors showed that the conserved WSXWS motif of the D2 domain undergoes conformational changes upon ligand binding (from a T-stack to a ladder) which serves as a molecular switch for activation. Consistently, the constitutively active PRLR-I146L showed a change in the dynamic of this motif.

The article of Tallet et al. had an important impact in its field as it allowed to exclude one hypothesis and definitely oriented the search for intramolecular conformational changes without impact on receptor stoichiometry or major changes in the distance of the extracellular domains. Future work will now fully concentrate on the mechanism of PRLR activation involving intramolecular conformational changes. Recent advances on the activation mechanism of other cytokine receptors such as the growth hormone receptor (GHR) appear to consolidate the proposed PRLR activation model and is likely to provide additional insights (8). According to the current model, GHR exists as preformed inactive dimer in which the two subunits are held together through weak interactions in the transmembrane domain. Receptor dimers are proposed to associate with a JAK2 dimer through the Box 1 motif in the intracellular region of the receptor in a way that the kinase domain of one JAK2 molecule binds to the pseudokinase domain of the other JAK2 molecule in "trans," thus inhibiting kinase activity in the basal state. Binding of growth hormone to GHR alters the position of the extracellular juxtamembrane, transmembrane, and intracellular domain of the GHR in an outward movement aligning and activating the two JAK2 kinase domains. Future studies will show whether PRLR activation fits to this model. Such a mechanism opens also new therapeutic routes. For example, antibodies directed against the extracellular juxtamembrane domain might stabilize the active conformation of the receptor or disruption of the JAK2 kinase/pseudokinase interaction with small molecules might help to activate the kinase.

\section{REFERENCES}

1. Tallet E, Fernandez I, Zhang C, Salsac M, Gregor N, Ayoub MA, et al. Investigation of prolactin receptor activation and blockade using time-resolved fluorescence resonance energy transfer. Front Endocrin (2011) 2:29. doi:10.3389/fendo.2011.00029

2. Qazi AM, Tsai-Morris CH, Dufau ML. Ligandindependent homo- and heterodimerization of human prolactin receptor variants: inhibitory action of the short forms by heterodimerization. Mol Endocrinol (2006) 20:1912-23. doi:10.1210/me. 2005-0291

3. Gadd SL, Clevenger CV. Ligand-independent dimerization of the human prolactin receptor isoforms: functional implications. Mol Endocrinol (2006) 20:2734-46. doi:10.1210/me.2006-0114

4. Tan D, Johnson DA, Wu W, Zeng L, Chen YH, Chen WY, et al. Unmodified prolactin (PRL) and
S179D PRL-initiated bioluminescence resonance energy transfer between homo- and hetero-pairs of long and short human PRL receptors in living human cells. Mol Endocrinol (2005) 19:1291-303. doi:10.1210/me.2004-0304

5. Couturier C, Jockers R. Activation of leptin receptor by a ligand-induced conformational change of constitutive receptor dimers. J Biol Chem (2003) 278:26604-11. doi:10.1074/jbc.M302002200

6. Vauthier V, Derviaux C, Douayry N, Roux T, Trinquet $\mathrm{E}$, Jockers $\mathrm{R}$, et al. Design and validation of a homogeneous time-resolved fluorescence-based leptin receptor binding assay. Anal Biochem (2013) 436:1-9. doi:10.1016/j.ab.2012.12.013

7. Dagil R, Knudsen MJ, Olsen JG, O’Shea C, Franzmann M, Goffin V, et al. The WSXWS motif in cytokine receptors is a molecular switch involved in receptor activation: insight from structures of the prolactin receptor. Structure (2012) 20:270-82. doi:10.1016/j.str.2011.12.010

8. Brooks AJ, Dai W, O’Mara ML, Abankwa D, Chhabra Y, Pelekanos RA, et al. Mechanism of activation of protein kinase JAK2 by the growth hormone receptor. Science (2014) 344:1249783. doi:10.1126/ science. 1249783

Conflict of Interest Statement: The author declares that the research was conducted in the absence of any commercial or financial relationships that could be construed as a potential conflict of interest.

Received: 26 May 2014; accepted: 29 May 2014; published online: 18 June 2014.

Citation: Jockers $R$ (2014) Frontiers Commentary on Tallet et al. Investigation of prolactin receptor activation and blockade using time-resolved fluorescence resonance energy transfer. Front. Endocrinol. 5:88. doi: 10.3389/fendo.2014.00088

This article was submitted to the journal Frontiers in Endocrinology.

Copyright (c) 2014 Jockers. This is an open-access article distributed under the terms of the Creative Commons Attribution License (CC BY). The use, distribution or reproduction in other forums is permitted, provided the original author(s) or licensor are credited and that the original publication in this journal is cited, in accordance with accepted academic practice. No use, distribution or reproduction is permitted which does not comply with these terms. 